\title{
Minimum Mean-Square Error and Maximum Likelihood Multiuser Detection: Statistical Properties and Applications
}

\author{
Lie-Liang Yang \\ School of ECS, University of Southampton, SO17 1BJ, United Kingdom \\ Tel: 0044-(0)23-8059 3364, Email:1ly@ecs.soton.ac.uk, http://www-mobile.ecs.soton.ac.uk
}

\begin{abstract}
Although multiuser detection (MUD) has received intensive research since its invention by Verdu in 1983, however, the statistical properties in MUD have not received enough attention in research and application. In this contribution, we first study the statistical properties of minimum mean-square error (MMSE)-MUD, maximum likelihood (ML)MUD and hybrid MMSE/ML-MUD from different aspects in the context of direct-sequence code-division multiple-access (DS-CDMA) communicating over additive white Gaussian noise (AWGN) channels. Both the amplitude statistics and ratio statistics are investigated. Then, the applications of the statistics of MUD are discussed. Our study shows that the statistics embedded in the MUD schemes may be exploited for design of highefficiency search algorithms in ML-MUD, for design of good spreading sequences, for post-MUD processing enhancement, for design of novel MUD schemes, etc.
\end{abstract}

\section{INTRODUCTION}

Multiuser detection (MUD) has received intensive research, since its invention by Verdu in 1983 [1]. So far, many types of MUD schemes have been proposed and studied (see, e.g, [2-4]) and have been found a lot of applications in various communications scenarios, including typically code-division multiple-access (CDMA) systems as well as the other multiple-input multiple-output (MIMO) systems, where signals experience intersymbol interference (ISI), co-channel interference (CCI), multiuser interference (MUI), etc. However, the statistical properties of the various MUD schemes have received little attention in research, even though the MUD's outputs are often stated as Gaussian distributed, such as in zero-forcing (ZF)-MUD [2], or as approximately Gaussian distributed, such as in MMSE-MUD [5, 6], random variables.

Therefore, in this contribution we focus our attention on the statistical properties of MUD. Specifically, the statistics related to both the linear MMSE-MUD and nonlinear ML-MUD [2] are studied. The statistics of these two MUD schemes are observed from different aspects with our emphasis on finding their possible applications, in order to improve the efficiency of the corresponding communications systems. In this contribution both the amplitude statistics and ratio statistics, which will be defined in our forthcoming discourses, are addressed under various constraints. Finally, the applications of the statistical properties of MUD are discussed. As shown in Section V, the statistical properties of the MMSE-MUD and ML-MUD may be exploited for designing high-efficiency search algorithms in MLMUD, for designing good spreading sequences to achieve enhanced bit-error-rate (BER) performance, for enhancing post-MUD processing, for designing novel MUD schemes, etc. Specifically, as an example, in this contribution a hybrid MMSE/ML-MUD scheme is proposed and its BER performance is investigated by simulations. Our study shows that the hybrid MMSE/ML-MUD can be a high-efficiency MUD scheme, which is capable of providing a good trade-off between the affordable complexity and the achievable BER performance.

Note that, in this contribution our study considers only the MMSEMUD and ML-MUD for DS-CDMA systems communicating over AWGN channels. The extensions to the other communications scenarios are straightforward. First, they can be extended by invoking various MUD schemes, when AWGN or fading channels are considered. Second, the MUD's statistical properties may be investigated in the context of the other MIMO systems, where signals conflict ISI, CCI, MUI, etc.

\section{PRELIMinaries}

Consider a synchronous DS-CDMA system supporting $K$ number of users, which transmit binary phase-shift keying (BPSK) modulated signals over AWGN channels, the received discrete signals at the basestation (BS) can be written as [2]

$$
\boldsymbol{y}=\boldsymbol{C} \boldsymbol{b}+\boldsymbol{n}=\sum_{k=1}^{K} \boldsymbol{c}_{k} b_{k}+\boldsymbol{n}
$$

where $\boldsymbol{y}$ denotes a $N$-length observation vector, $\boldsymbol{C}=\left[\boldsymbol{c}_{1}, \boldsymbol{c}_{2}, \cdots, \boldsymbol{c}_{K}\right]$ is a $(N \times K)$ spreading matrix, which is constructed by the $N$-length spreading sequences assigned to the $K$ users, $\boldsymbol{c}_{k}=$ $\left[c_{k 0}, c_{k 1}, \cdots, c_{k(N-1)}\right]^{T} / \sqrt{N}$ is the $k$ th user's spreading sequence, where $c_{k n} \in\{+1,-1\}$ and $\left\|\boldsymbol{c}_{k}\right\|^{2}=1$. In (1) $\boldsymbol{b}=\left[b_{1}, b_{2}, \ldots, b_{K}\right]^{T}$ contains the data bits transmitted by the $K$ users, $b_{k}$ is assumed to be an independent binary random variable taking a value of +1 or -1 with equal probability. Finally, in (1) $n$ is a $N$-length Gaussian noise vector obeying the multivariate normal distribution associated with mean zero and a covariance matrix of $\sigma^{2} \boldsymbol{I}_{N}$, where $\sigma^{2}=\left(2 \gamma_{b}\right)^{-1}$ and $\gamma_{b}$ denotes the signal-to-noise ratio (SNR) per bit. It has been shown in [2] that the sufficient statistics for detection of $\boldsymbol{b}$ can be obtained as

$$
\overline{\boldsymbol{y}}=\boldsymbol{C}^{T} \boldsymbol{y}=\boldsymbol{R} \boldsymbol{b}+\overline{\boldsymbol{n}}
$$

where $R=C^{T} C$ denotes the auto-correlation matrix of $C$, while $\bar{n}=C^{T} n$ is now a $K$-length Gaussian noise vector distributed with mean zero and a covariance matrix $\sigma^{2} R$.

In the context of the optimum ML-MUD, the estimate to $\boldsymbol{b}$ can be obtained by solving any one of the following equivalent optimization problems $[2,4]$

$$
\begin{aligned}
\hat{\boldsymbol{b}} & =\arg \min _{\tilde{\boldsymbol{b}} \in\{+1,-1\}^{K}}\left\{\lambda(\tilde{\boldsymbol{b}})=\|\boldsymbol{y}-\boldsymbol{C} \tilde{\boldsymbol{b}}\|^{2}\right\} \\
& \triangleq \arg \min _{\tilde{\boldsymbol{b}} \in\{+1,-1\}^{K}}\left\{\lambda_{a}(\tilde{\boldsymbol{b}})=(\overline{\boldsymbol{y}}-\boldsymbol{R} \tilde{\boldsymbol{b}})^{T} \boldsymbol{R}^{-1}(\overline{\boldsymbol{y}}-\boldsymbol{R} \tilde{\boldsymbol{b}})\right\} \\
& \triangleq \arg \min _{\tilde{\boldsymbol{b}} \in\{+1,-1\}^{K}}\left\{\lambda_{b}(\tilde{\boldsymbol{b}})=\tilde{\boldsymbol{b}}^{T} \boldsymbol{R} \tilde{\boldsymbol{b}}-2 \overline{\boldsymbol{y}}^{T} \tilde{\boldsymbol{b}}\right\}
\end{aligned}
$$

where $\tilde{\boldsymbol{b}}$ is a $K$-length binary test vector. Note that, the condition for using (4) is that the auto-correlation matrix $R$ is invertible, which often implies that $K \leq N$. By contrast, there is no such constraint for using (3) and (5). Explicitly, the optimum ML-MUD is a nonlinear MUD and requires an exhaustive search over all the $2^{K}$ possible combinations of the components in the binary data vector $\boldsymbol{b} \in\{+1,-1\}^{K}$, in order to find the optimum solution to $b$. Hence, the computational complexity of the ML-MUD is exponentially dependent on the number of users of $K$, making the optimum ML-MUD impractical to implement, when the number of users supported is relatively high. For this sake, efficient suboptimum algorithms, such as those summarized in [4], have usually been proposed for finding near-optimum solutions for the ML-MUD, yielding the suboptimum ML-MUDs.

Furthermore, in order to improve the efficiency of the suboptimum ML-MUDs, the detection process may be started with a lowcomplexity linear single-user or linear MUD procedure. By doing this, the detector has obtained certain amount of information about the transmitted bits before starting the suboptimum ML-MUD procedure, which may hence assist to find the near-optimum solution for the 
suboptimum ML-MUD. In this contribution, as an example, the linear MMSE-MUD is invoked, which carries out the detection by forming a decision variable vector $\boldsymbol{z}=\left[z_{1}, z_{2}, \cdots, z_{K}\right]^{T}$ as

$$
\boldsymbol{z}=\boldsymbol{W}^{T} \boldsymbol{y}
$$

where $W$ is the optimum weight matrix in MMSE sense, which can be expressed as $[2,4]$

$$
\boldsymbol{W}=\left(\boldsymbol{C} \boldsymbol{C}^{T}+\sigma^{2} \boldsymbol{I}_{N}\right)^{-1} \boldsymbol{C}
$$

where $\boldsymbol{R}_{y}=\left(\boldsymbol{C} \boldsymbol{C}^{T}+\sigma^{2} \boldsymbol{I}_{N}\right)$ is the auto-correlation matrix of the observation vector $\boldsymbol{y}$. It has been shown [5] that, after the MMSEMUD, the decision variables $z_{k}, k=1, \ldots, K$ can be closely approximated as independent Gaussian random variables expressed as

$$
z_{k}=b_{k}+\tilde{n}_{k}, k=1,2, \ldots, K
$$

where $\tilde{n}_{k}$ is Gaussian distributed with mean zero and a variance given by $[4,5]$

$$
\tilde{\sigma}^{2}=\left[\left(1-\boldsymbol{c}_{k}^{T} \boldsymbol{R}_{y}^{-1} \boldsymbol{c}_{k}\right)^{-1}-1\right]^{-1}
$$

Let us now study the statistics, when various detection scenarios are considered.

\section{Amplitude Statistics}

In this section we study the amplitude statistics of the ML-metric $\lambda(\cdot)$ in (3) and the decision variable $z_{k}, k=1, \ldots, K$, of (8). The PDFs depicted are either evaluated from the formulas provided or obtained by simulations. Let us first consider the amplitude statistics related to the ML-MUD.

\section{A. Maximum Likelihood Multiuser Detection}

1) Probability Distribution of $\lambda(\boldsymbol{b})$ : Letting $\tilde{\boldsymbol{b}}=\boldsymbol{b}$, from (1) and (3) we know that

$$
\lambda(\boldsymbol{b})=\|\boldsymbol{y}-\boldsymbol{C b}\|^{2}=\|\boldsymbol{n}\|^{2}
$$

Since $\boldsymbol{n}$ is a multivariate Gaussian random vector distributed with mean zero and a covariance matrix $\sigma^{2} \boldsymbol{I}_{N}$, it can be shown that the normalized ML-metric $\bar{\lambda}(\boldsymbol{b})=\lambda(\boldsymbol{b}) / \sigma^{2}$ obeys the central $\chi^{2}$-distribution of $N$ degrees-of-freedom with the probability density function (PDF) given by $[7,8]$

$$
f_{\bar{\lambda}(\boldsymbol{b})}(y)=\frac{1}{2 \Gamma(N / 2)}\left(\frac{y}{2}\right)^{N / 2-1} \exp \left(-\frac{y}{2}\right), y \geq 0
$$

where $\Gamma(\cdot)$ is the gamma function [7].

2) Probability Distribution of $\lambda\left(\boldsymbol{b}^{(U)}\right)$ : Let $\boldsymbol{b}^{(U)}$ denote a $K$-length test vector having $U \leq K$ differences from the transmitted data vector $\boldsymbol{b}$ as seen in (1). Then, the ML-metric $\lambda\left(\boldsymbol{b}^{(U)}\right)$ is given by

$$
\lambda\left(\boldsymbol{b}^{(U)}\right)=\left\|\boldsymbol{y}-\boldsymbol{C b}^{(U)}\right\|^{2}=\left\|\boldsymbol{C}\left(\boldsymbol{b}-\boldsymbol{b}^{(U)}\right)+\boldsymbol{n}\right\|^{2}
$$

It can be shown that, when assuming that $C$ is a random binary spreading matrix, the PDF of $\lambda\left(\boldsymbol{b}^{(U)}\right)$ can be obtained. However, this PDF has a complicated structure, and furthermore, it is very hard to evaluate, when the value of $U$ is high. For this sake, Monte-Carlo based simulation approaches are applied to obtain the PDF of $\lambda\left(\boldsymbol{b}^{(U)}\right)$ or of its normalized version $f_{\bar{\lambda}(\boldsymbol{b}(U))}(y)$.

Fig. 1 illustrates the probability distributions of the ML-metrics in the DS-CDMA systems using random spreading sequences, when the test vector $\tilde{\boldsymbol{b}}$ has $U=0,1,2$ or 3 differences from the transmitted data vector $\boldsymbol{b}$. Note that, the reason for us to consider only the cases of $U=$ $0,1,2$ and 3 is that their PDFs can provide us important information about the convergence behaviour of the ML-MUD. The PDFs in Fig. 1 show that the distribution curve moves toward the righthand-side as the number of differences between the test vector $\tilde{b}$ and the transmitted vector $\boldsymbol{b}$ increases. Hence, when the test vector $\tilde{b}$ has more differences

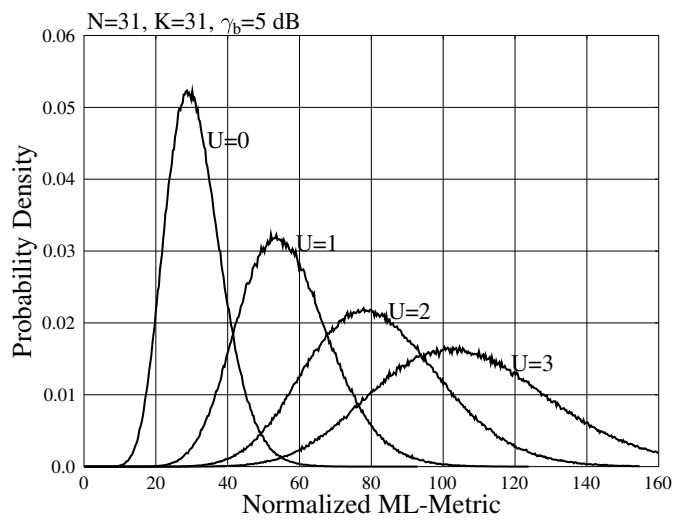

Fig. 1. Probability distribution of the ML-metrics in the DS-CDMA systems using random spreading sequences, when the test vector $\tilde{\boldsymbol{b}}$ has $U=0,1,2$ or 3 differences from the transmitted vector $\boldsymbol{b}$.

from the transmitted vector $\boldsymbol{b}$, it generally results in a higher ML-metric value.

Additionally, according to the statistics of the ML-MUD, we know that the number of $K$-length binary vectors having low differences, such as $U=0,1,2$, etc., from the transmitted vector is small. Hence, when a sufficiently low ML-metric appears during a random search process, it is then highly confident that the corresponding test vector $\tilde{\boldsymbol{b}}$ is the actual vector $\boldsymbol{b}$ transmitted or close to the vector $\boldsymbol{b}$ transmitted. Therefore, the distribution information of the ML-metrics may be exploited for improving the efficiency of search in ML-MUD.

\section{B. Minimum Mean-Square Error Multiuser Detection}

Given the decision variables as shown in (8), if $\left\{z_{k}\right\}$ can be approximated as independent identically distributed (iid) Gaussian random variables, the reliabilities of the detected bits in $\hat{b}$ based on $\left\{z_{k}\right\}$ can be measured by the amplitudes of $\left\{\left|z_{k}\right|\right\}$ or the normalized amplitudes $\left\{\left|\bar{z}_{k}\right|=\left|z_{k}\right| / \tilde{\sigma}\right\}$ [9]. Let us define the following variables:

$$
A_{i}=\min _{i}\left\{\left|\bar{z}_{1}\right|,\left|\bar{z}_{2}\right|, \ldots,\left|\bar{z}_{K}\right|\right\}, i=1,2, \ldots, K
$$

where $A_{i}$ represents the reliability of the $i$ th-most unreliable bit in $\hat{\boldsymbol{b}}$. We have $A_{1} \leq A_{2} \leq \ldots \leq A_{K} ; A_{1}$ and $A_{K}$ are the reliabilities of the most unreliable and most reliable bits in $\hat{b}$, respectively.

1) Probability Distribution of $A_{i}$ : The PDF of $A_{i}, i=1, \ldots, K$ can be derived, which can be expressed as

$$
\begin{aligned}
f_{A_{i}}(y)= & \sum_{u=0}^{i-1} \sum_{v=0}^{K-i}(-1)^{i-u-1} \frac{K !}{u !(i-u-1) ! v !(K-i-v) !} \\
& \times Q^{u}(\sqrt{2 \tilde{\gamma}}-y) Q^{v}(y-\sqrt{2 \tilde{\gamma}}) \\
& \times[Q(\sqrt{2 \tilde{\gamma}}+y)]^{K-u-v-1} f_{\left|\bar{z}_{k}\right|}(y)
\end{aligned}
$$

where $\tilde{\gamma}=1 / 2 \tilde{\sigma}^{2}, Q(x)$ is the Gaussian $Q$-function defined as $Q(x)=(2 \pi)^{-1 / 2} \int_{x}^{\infty} e^{-t^{2} / 2} d t$, and $f_{\left|\bar{z}_{k}\right|}(y)$ is given by

$$
f_{\left|\bar{z}_{k}\right|}(y)=\frac{1}{\sqrt{2 \pi}}\left[e^{-\frac{(y-\sqrt{2 \tilde{\gamma}})^{2}}{2}}+e^{-\frac{(y+\sqrt{2 \bar{\gamma}})^{2}}{2}}\right], y \geq 0
$$

Fig. 2 shows the statistics of the ordered reliabilities of the MMSEMUD in a DS-CDMA system employing a spreading factor $N=31$ and supporting $K=31$ users, when communicating over AWGN channels. It can be observed that, after the MMSE-MUD, the reliabilities of the detected data bits are highly sparse, some of them are much more reliable than the others. As shown in our forthcoming discourse, the reliability information extracted from the MMSE-MUD may be 


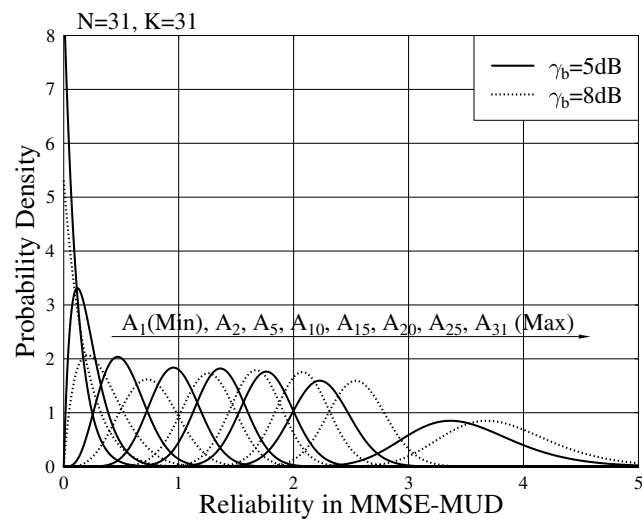

Fig. 2. Probability distribution of the ordered reliabilities of the MMSE-MUD for a DS-CDMA system using random spreading sequences.

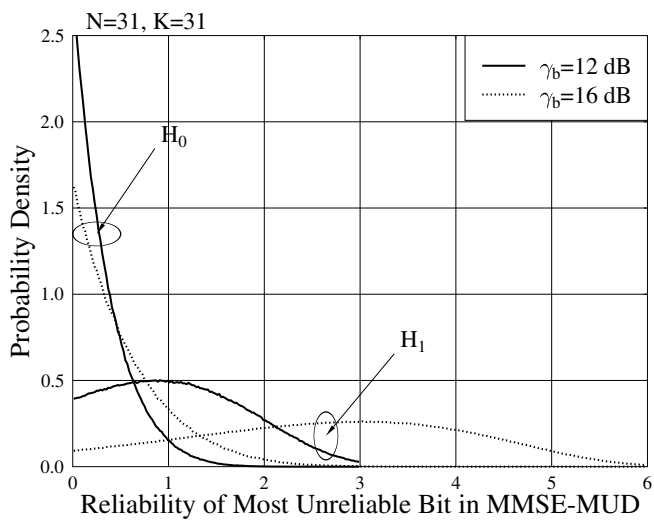

Fig. 3. PDF of reliability of the most unreliable bit on condition that the MMSE-MUD is correct $\left(H_{1}\right)$ or incorrect $\left(H_{0}\right)$, for a DS-CDMA system using random spreading sequences.

exploited for significantly improving the BER performance of the DSCDMA systems.

2) Probability Distribution of $A_{1}$ When MMSE-MUD Is Correct/Incorrect: Let us denote $H_{1}$ the event that the MMSE-MUD is correct and $H_{0}$ the event that the MMSE-MUD is incorrect. Then, $f_{A_{1}}\left(y \mid H_{1}\right)$ and $f_{A_{1}}\left(y \mid H_{0}\right)$ represent the PDFs of the reliabilities of the most unreliable bit in $\hat{\boldsymbol{b}}$, on condition that the detection of $\hat{\boldsymbol{b}}$ by the MMSE-MUD is correct and incorrect, respectively. In Fig. 3 these two PDFs are drawn for a DS-CDMA system using a spreading factor of $N=31$ and supporting $K=31$ users at a SNR of $\gamma_{b}=12 \mathrm{~dB}$ or $16 \mathrm{~dB}$. As seen in Fig. 3, the two PDFs conditioned on the correct and incorrect MMSE-MUD are distinct, implying that the minimum amplitude $A_{1}$ of the decision variables generated by the MMSE-MUD is capable of providing the receiver information about whether the corresponding detection is reliable or unreliable. As shown in Fig. 3 and considering SNR of $\gamma_{b}=16 \mathrm{~dB}$, if the receiver measures that the minimum amplitude of $A_{1}$ is higher than 2.5 , it then has a very high confidence that the corresponding detection is correct. By contrast, if it is found that the minimum amplitude of $A_{1}$ is lower than 1.0, then, the corresponding detection may be in error with a high probability.

C. Hybrid Minimum Mean-Square Error Maximum-Likelihood Multiuser Detection

From Sections III-A and III-B, we are implied that the reliability information provided by the MMSE-MUD may be exploited by the ML-MUD, in order to achieve a better BER performance than the

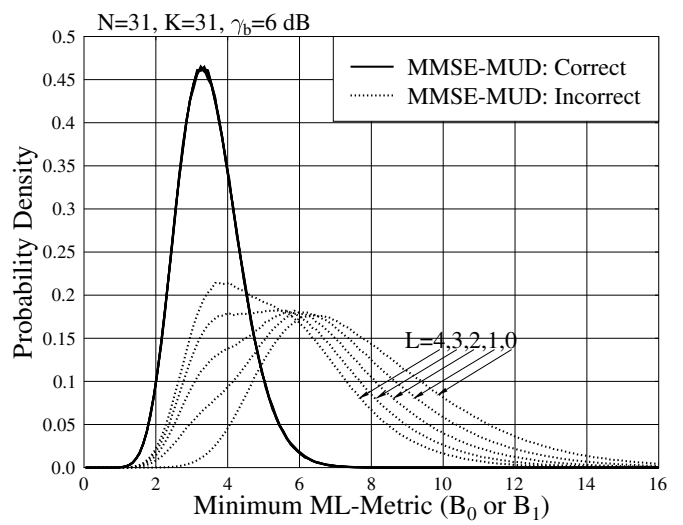

Fig. 4. PDF of the minimum ML-metric $B_{0}$ when $L=0$ or $B_{1}$ when $L>0$, on condition that the MMSE-MUD is correct $\left(H_{1}\right)$ or incorrect $\left(H_{0}\right)$.

MMSE-MUD, while with a complexity significantly lower than that of the ML-MUD. For convenience, we refer to this MUD scheme as the hybrid MMSE/ML-MUD. In this subsection we study the related statistics of the hybrid MMSE/ML-MUD. Its BER performance will be provided in Section V.

Let after the MMSE-MUD $\hat{\boldsymbol{b}}_{i}=\left[\hat{b}_{i_{1}}, \hat{b}_{i_{2}}, \ldots, \hat{b}_{i_{L}}\right]^{T}$ contains the $L(0 \leq L \leq K)$ number of most unreliable bits, while the other $(K-L)$ bits are assumed to have been detected reliably by the MMSEMUD. Explicitly, $L=0$ corresponds to the case that the detector accepts the MMSE-MUD's estimation as the final estimation, while $L=K$ corresponds the scenario that the detector ignores the MMSEMUD's estimation and carries out the complete ML-MUD. Letting $\hat{b}_{i_{l}}, l=1, \ldots, L$ takes values randomly in $\{+1,-1\}$, we can generate $M=2^{L}$ ML-metrics, which can be expressed in the form as

$$
\left\{Z_{m}\right\}=\left\{\left\|\boldsymbol{y}-\boldsymbol{C b}\left(\hat{\boldsymbol{b}}_{i}\right)\right\|^{2}: \hat{\boldsymbol{b}}_{i} \in\{+1,-1\}^{L}\right\}
$$

where $\boldsymbol{b}\left(\hat{\boldsymbol{b}}_{i}\right)$ denotes a $K$-length binary test vector constructed by the bits assumed to have been reliably detected and the bits from one realization of $\hat{\boldsymbol{b}}_{i} \in\{+1,-1\}^{L}$. Let the $n$ th-minimum of $\left\{Z_{1}, Z_{2}, \ldots, Z_{M}\right\}$ be expressed as

$$
B_{n}=\min _{n}\left\{Z_{1}, Z_{2}, \ldots, Z_{M}\right\}, n=1,2, \ldots, M
$$

Additionally, we can see that, when $L=0$, there is only one MLmetric, which is defined as $B_{0}=Z_{1}$, where $Z_{1}$ is obtained by letting the test vector in ML-MUD equal to the MMSE-MUD's estimated vector. Below we consider the statistics of $B_{0}$ or $B_{1}$, when the MMSEMUD is correct (event $H_{1}$ ) or incorrect (event $H_{0}$ ), or when the MLMUD is correct (event $\mathcal{H}_{1}$ ) or incorrect (event $\mathcal{H}_{0}$ ).

Fig. 4 shows the PDF of the minimum ML-metric of $B_{0}$ when $L=0$ and $B_{1}$ when $L>0$, on condition that the MMSE-MUD is correct $\left(H_{1}\right)$ or incorrect $\left(H_{0}\right)$. By contrast, in Fig. 5 the PDF of the minimum ML-metric of $B_{0}$ when $L=0$ and $B_{1}$ when $L>0$ is drawn, on condition that the ML-MUD is correct $\left(\mathcal{H}_{1}\right)$ or incorrect $\left(\mathcal{H}_{0}\right)$. For both figures the DS-CDMA systems using random spreading sequences were assumed, and the DS-CDMA systems were full-load corresponding to $K=N=31$. As shown in Figs. 4 and 5, if the detection of the MMSE-MUD or the ML-MUD is correct, the distributions of $B_{0}$ and $B_{1}$ for different values of $L$ retain nearly the same. This observation implies that, when the detection of the MMSEMUD or ML-MUD is correct, the minimum of $\left\{Z_{m}\right\}$ generally follows the distribution of the ML-metric generated directly using the transmitted data vector. However, when the detection of the MMSEMUD or ML-MUD is incorrect, as shown in Figs. 4 and 5, the PDF of $B_{1}$ of the minimum ML-metric in $\left\{Z_{m}\right\}$ is different for different $L$ values. Furthermore, as shown in Fig. 4 , when the value of $L$ 


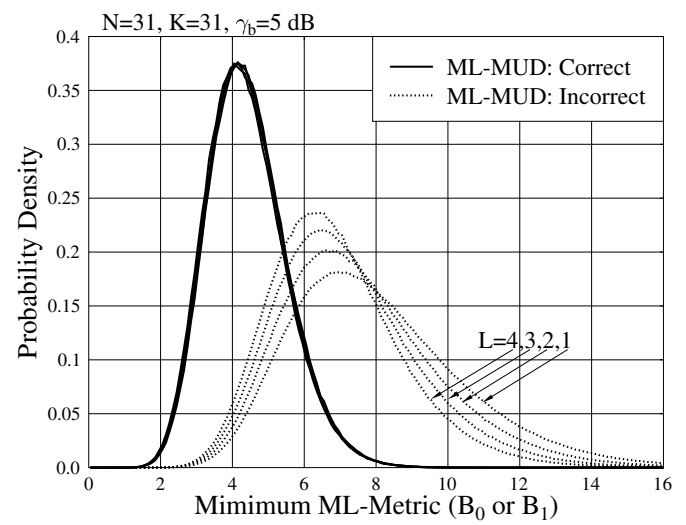

Fig. 5. PDF of the minimum ML-metric $B_{0}$ when $L=0$ or $B_{1}$ when $L>0$, on condition that the ML-MUD is correct $\left(\mathcal{H}_{1}\right)$ or incorrect $\left(\mathcal{H}_{0}\right)$.

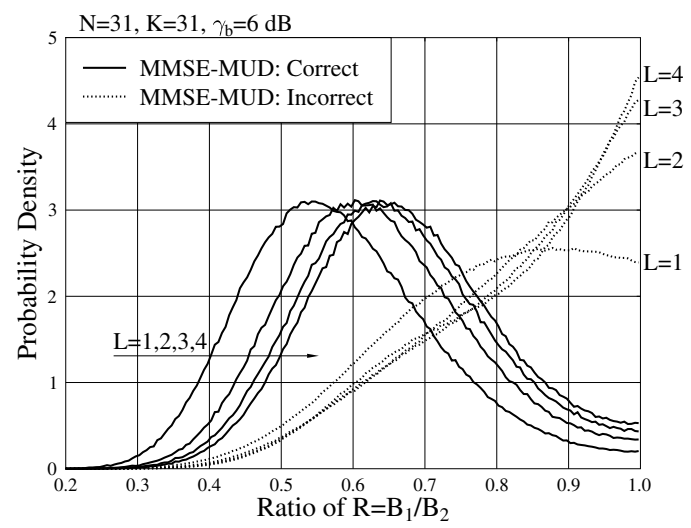

Fig. 6. PDF of the ratio of $R=B_{1} / B_{2}$ on condition that the MMSE-MUD is correct $\left(H_{1}\right)$ or incorrect $\left(H_{0}\right)$ for a DS-CDMA system using random spreading sequences.

increases, the distribution of $B_{1}$ on condition that the MMSE-MUD is incorrect becomes close to and tends to resemble the distribution of $B_{1}$ on condition that the MMSE-MUD is correct. This is because, even although the MMSE-MUD is incorrect, the minimum ML-metric $B_{1}$ of $\left\{Z_{m}\right\}$ might still be the one generated by the transmitted data vector. In this case, the ML-MUD produces correct detection.

\section{RATIO STATISTICS}

In this section the ratio statistics is studied in the context of the hybrid MMSE/ML-MUD. The ratio concerned is defined as

$$
R=\frac{B_{1}}{B_{2}}
$$

where $B_{1}$ and $B_{2}$ are defined by (17), $B_{1}$ represents the minimum of $\left\{Z_{m}\right\}$, while $B_{2}$ represents the second minimum of $\left\{Z_{m}\right\}$.

Straightforwardly, if the detection of the MMSE-MUD or ML-MUD is correct, then, the minimum $B_{1}$ is generated by a test vector that is the same as the transmitted vector $\boldsymbol{b}$, but the second minimum $B_{2}$ is generated by a test vector different from the transmitted vector $\boldsymbol{b}$. In this case, according to Fig. 1 , the ratio $R=B_{1} / B_{2}$ will be significantly lower than one. By contrast, if the detection of the MMSE-MUD or ML-MUD is incorrect, then, the minimum $B_{1}$, the second minimum $B_{2}$, and even $B_{3}, B_{4}$, etc. may be similar. In this case, the ratio $R=B_{1} / B_{2}$ will be close to one.

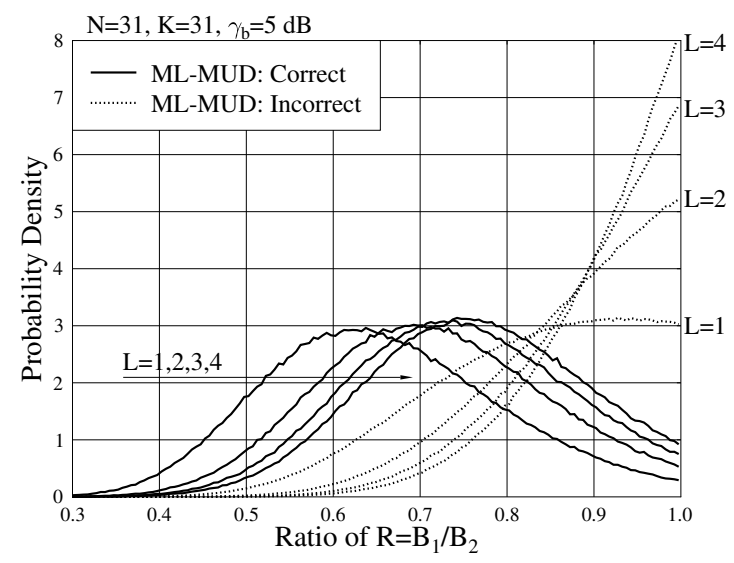

Fig. 7. PDF of the ratio of $R=B_{1} / B_{2}$ on condition that the ML-MUD is correct $\left(\mathcal{H}_{1}\right)$ or incorrect $\left(\mathcal{H}_{0}\right)$ for a DS-CDMA system using random spreading sequences.

Therefore, in Fig. 6 we illustrate the PDF of $R=B_{1} / B_{2}$ on condition that the detection of the MMSE-MUD is correct $\left(H_{1}\right)$ or incorrect $\left(H_{0}\right)$, while in Fig. 7 the PDF of $R=B_{1} / B_{2}$ on condition that the detection of the ML-MUD is correct $\left(\mathcal{H}_{1}\right)$ or incorrect $\left(\mathcal{H}_{0}\right)$. It is observed that the ratio $R=B_{1} / B_{2}$ is mainly distributed in the region close to one, when the detection of the MMSE-MUD or the MLMUD is incorrect. Otherwise, when the detection of the MMSE-MUD or the ML-MUD is correct, the ratio $R=B_{1} / B_{2}$ is then distributed within a region having the ratio values explicitly lower than one.

Let us below discuss some possible applications inspired by the statistical properties of the MMSE- and ML-MUD.

\section{APPLICATION EXAMPLES}

When the statistical properties related to the MMSE-MUD or/and ML-MUD are available, they may be exploited for enhancing the performance of the corresponding DS-CDMA system in various ways. Below are some examples showing the possible applications.

\section{A. Design of Efficient Search Algorithms}

For example, when the statistics of $\lambda\left(\boldsymbol{b}^{(U)}\right)$ or $\bar{\lambda}\left(\boldsymbol{b}^{(U)}\right)$ for $u=$ $0,1, \ldots, K$ are available, i.e., when the receiver knows the PDFs of $f_{\lambda(\boldsymbol{b}(U))}(y)$ or $f_{\bar{\lambda}(\boldsymbol{b}(U))}(y)$ for $u=0,1, \ldots, K$, then, given a ML-metric $\lambda$ or $\bar{\lambda}$, the receiver may obtain certain information about how many differences between the corresponding test vector and the transmitted vector $\boldsymbol{b}$. Consequently, the ML-MUD may improve its search efficiency with the aid of this information.

\section{B. Design of Spreading Sequences}

Assuming a ML test vector $\boldsymbol{b}^{(U)}$, which has $U$ differences from the transmitted data vector $\boldsymbol{b}$. Then, according to (12) and when ignoring the background noise, the ML-metric is given by

$$
\lambda\left(\boldsymbol{b}^{(U)}\right)=\left\|\boldsymbol{y}-\boldsymbol{C b}^{(U)}\right\|^{2}=\left\|\boldsymbol{C}\left(\boldsymbol{b}-\boldsymbol{b}^{(U)}\right)\right\|^{2}
$$

This equation implies that $\lambda\left(\boldsymbol{b}^{(U)}\right)$ for $U \neq 0$ should be as large as possible in order to minimize the error probability after the ML-MUD. This observation can also be reflected by Fig. 1: in order for the MLMUD to achieve the minimum error probability, the overlapping area between the PDF of $f_{\bar{\lambda}(\boldsymbol{b}(0))}(y)$ and the other PDFs of $f_{\bar{\lambda}(\boldsymbol{b}(U))}(y)$ for $U \neq 0$ should be as low as possible. Therefore, an optimum set of spreading sequences for the DS-CDMA system using ML-MUD should be designed according to

$$
\boldsymbol{C}_{\text {opt }}=\arg \max _{\boldsymbol{C}}\left\{\lambda\left(\boldsymbol{b}^{(U)}\right)=\left\|\boldsymbol{C}\left(\boldsymbol{b}-\boldsymbol{b}^{(U)}\right)\right\|^{2}\right\}
$$




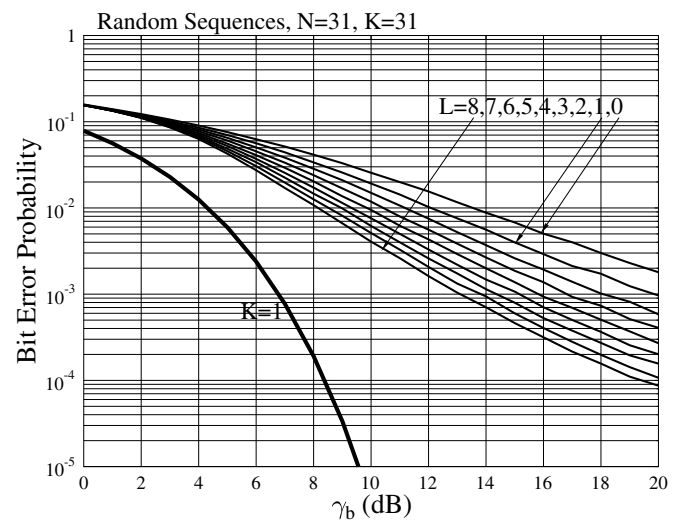

Fig. 8. BER versus SNR per bit $\gamma_{b}$ performance of the DS-CDMA systems using conventional MMSE-MUD $(L=0)$ and hybrid MMSE/ML-MUD, when random spreading sequences are employed.

for any $U \neq 0$.

Note that, the optimization problem of (20) may be extremely hard to solve. For this sake, sub-optimum solutions may be obtained by considering the following facts. First, the PDFs $f_{\bar{\lambda}\left(\boldsymbol{b}^{(0)}\right)}(y)$ and $f_{\bar{\lambda}\left(\boldsymbol{b}^{(1)}\right)}(y)$ maintain the same for any set of spreading sequences. Second, from Fig. 1 we can be implied that only those test vectors having a small number of differences from the transmitted vector may have noticeable effect on the achievable error performance. Hence, the spreading sequences may be designed by considering only some low values of $U$, such as $U=2$ and 3 .

\section{Enhancing Post-MUD Processing}

The statistics obtained after the MMSE-MUD or ML-MUD can be exploited for enhancing the post-MUD processing. For example, with the aid of the reliability information provided by the MMSE-MUD or ML-MUD, in an error-control coding assisted DS-CDMA system softinput soft-output (SISO) decoding or iterative decoding [10] may be carried out in order to improve the system performance. Additionally, based on the reliability information, some unreliably detected data bits may be erased. Then, errors-and-erasure decoding can be carried out by the DS-CDMA receiver. It has been illustrated that the errors-anderasures decoding usually outperforms the errors-only decoding [11, 12].

\section{Design of Novel Multiuser Detection Schemes}

With the aid of the statistical properties of the multiuser detection, such as that of the MMSE-MUD and ML-MUD, novel MUD schemes may be designed. For example, a hybrid MMSE/ML-MUD scheme may be designed, which first carries out the MMSE-MUD. Then, as described in Section III-C, $L$ number of most unreliably detected bits are identified, which are finally replaced by the bits detected by the ML-MUD. Note that, the bits detected by the ML-MUD are contained in $\hat{\boldsymbol{b}}_{i}$, as seen in (16), which results in the minimum $B_{1}$ of $\left\{Z_{m}\right\}$.

Figs. 8 and 9 depict the BER performance of the DS-CDMA systems using hybrid MMSE/ML-MUD, when the DS-CDMA systems are full-load. The DS-CDMA considered in Fig. 8 employs random spreading sequences, while that in Fig. 9 employs Gold-sequences. As shown in Figs. 8 and 9, in comparison with the conventional MMSEMUD corresponding to $L=0$, the BER performance of the DSCDMA systems improves, when the value of $L$ increases, implying that the MUD involves a higher level of ML-MUD. As shown in Fig. 8, whenever the search space is doubled, there is some SNR gain. Specifically, the SNR gain for $L=8$ is more than $8 \mathrm{~dB}$ at the BER of $10^{-3}$, when comparing with the conventional MMSE-MUD. Therefore, the hybrid MMSE/ML-MUD is capable of providing a good

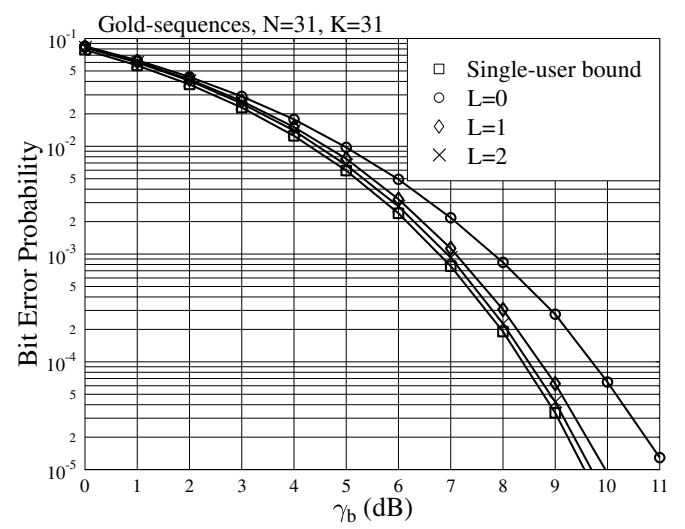

Fig. 9. BER versus SNR per bit $\gamma_{b}$ performance of the DS-CDMA systems using conventional MMSE-MUD $(L=0)$ and hybrid MMSE/ML-MUD, when Gold-sequences are employed.

trade-off between the affordable complexity and the achievable BER performance.

As shown in Fig. 9, when the DS-CDMA using Gold-sequences, the hybrid MMSE/ML-MUD is capable of achieving nearly the same BER performance as the conventional ML-MUD, when $L=2$. However, in this case the complexity of the hybrid MMSE/ML-MUD is significantly lower than that of the conventional ML-MUD. This is because the hybrid MMSE/ML-MUD only needs to search a space of size 4, while the conventional ML-MUD needs to search a space of size $2^{31}$.

In Summary, in this paper we have studied a range of statistics in the MMSE-MUD, ML-MUD and the hybrid MMSE/ML-MUD. It can be shown that these statistics may be found many applications for improving the performance of DS-CDMA systems.

Finally, note that, the work considered in this paper may be extended to various communications scenarios in wireless communications, including a variety of MIMO systems requiring MUD or equalization for suppressing ISI, CCI, MUI, etc.

\section{REFERENCES}

[1] S. Verdu, "Minimum probability of error for asychronous multiple access communication systems," in IEEE Mil. Comm. Conf.. IEEE, Oct. 1983, pp. 213-219.

[2] , Multiuser Detection. Cambridge University Press, 1998

[3] X. Wang and H. V. Poor, Wireless Communication Systems - Advanced Techniques for Signal Reception. Prentice Hall, 2003.

[4] L.-L. Yang, Multicarrier Communications. Chichester, United Kingdom: John Wiley, 2009.

[5] U. Madhow and M. L. Honig, "MMSE interference suppression for directsequence spread-spectrum CDMA," IEEE Trans. on Comm., vol. 42, no. 12 , pp. 3178-3188, Dec. 1994.

[6] H. V. Poor and S. Verdu, "Probability of error in MMSE multiuser detection," IEEE Trans. on Inf. Theory, vol. 43, no. 3, pp. 858-871, May 1997.

[7] J. G. Proakis, Digital Communications, 3rd ed. McGraw Hill, 1995.

[8] M. K. Simon, Probability Distributions Involving Gaussian Random Variables. Springer, 2006.

[9] J. Hagenauer, E. Offer, and L. Papke, "Iterative decoding of binary block and convolutional codes," IEEE Trans. on Inf. Theory, vol. 42, no. 2, pp. 429-445, Mar. 1996

[10] S. Lin and D. Costello, Error Control Coding: Fundamentals and Applications, 2nd ed. Upper Saddle River, NJ: Prentice-Hall, 1983.

[11] L.-L. Yang and L. Hanzo, "Performance analysis of coded M-ary orthogonal signaling using errors-and-erasures decoding over frequency-selective fading channels," IEEE J. on Select. Areas in Comm., vol. 19, no. 2, Feb. 2001 .

[12] L. L Yang and L. Hanzo, "Low complexity erasure insertion in RS-coded SFH spread-spectrum communications with partial-band interference and Nakagami-m fading," IEEE Trans. on Comm., vol. 50, no. 6, pp. 914-925, June 2002 . 